\title{
Minocycline for the treatment of Acinetobacter spp. infection
}

\author{
Authors \\ Maitreyi Bandyopadhyay ${ }^{1}$, Simit Kumar ${ }^{2 *}$, Manas Kumar Bandyopadhyay ${ }^{3}$, \\ Prabir Kumar Mukhopadhyay ${ }^{4}$, Anindita Chatterjee ${ }^{5}$, Mitali Chatterjee ${ }^{6}$ \\ ${ }^{1,2}$ Associate Professor, Dept. of Microbiology, R.G.Kar Medical College, Kolkata \\ ${ }^{3,4,6}$ Professor, Dept. of Microbiology, R.G.Kar Medical College, Kolkata \\ ${ }^{5}$ Research Associate, Dept. of Microbiology, R.G.Kar Medical College, Kolkata \\ Corresponding Author* \\ Dr Simit Kumar \\ Associate Professor, Dept of Microbiology, R.G.Kar Medical College, Kolkata \\ Email:simitkumar@gmail.com, Mobile No: 9339485642
}

\begin{abstract}
Background: Acinetobacter species have been increasingly recognized as a major pathogen implicated in hospital-acquired and healthcare-associated infections worldwide. With the increasing incidence of Carbapenem resistance, the other alternative, Colistin retains susceptibility but issues of appropriate dosing, toxicities, and resistance remain. Minocycline an old tetracycline, with high lipophilicity, high tissue penetration, long half life, good oral bioavailability, no serious side effects and the ability to be used in combinations when monotherapy fails, has been found to be an effective alternative therapy for MDR Acinetobacter associated infections.

Aims \& Objectives: To determine the activity of tetracycline group of drugs against Acinetobacter species.

Result: A total of 300 Acinetobacter species were included in this study of which $61.7 \%$ were carbapenem resistant isolates, $59.3 \%$ were multi-drug resistant Acinetobacter spp. Acinetobacter spp simultaneously resistant to carbapenems, fluoroquinolones and beta-lactams were defined as MDR in our study. Majority of isolates were sensitive to polymixin B (95\%), minocycline (67.3\%) and tigecycline (78\%). Tigecycline was the second most effective antibiotic after Colistin, followed by minocycline. Out of the 10 strains which were resistant to Colistin as well as tigecycline, the only other effective antibiotic was Minocycline (7/10).

Discussion: Tigecycline was the second most active isolate following Colistin, but most of the isolates in the study were from blood culture and pulmonary sites, where the activity of tigecycline remains doubtful. Minocycline thus theoretically appears to be the second most active agent. With the pharmacokinetic and pharmacodynamic advantages of minocycline and its ability to act in combination with colistin at half the dosage, it turns out to be a useful alternative for the institution of empirical therapy, followed by the monotherapy if proven to be culture sensitive, or combination can be considered when culture resistant, followed by oral therapy in cases of MDR Acinetobacter infections.

Keywords: Acinetobacter baumannii, MDR, minocycline.
\end{abstract}




\section{Introduction}

Acinetobacter species have been increasingly recognized as a major pathogen implicated in hospital-acquired and healthcare-associated infections worldwide. The common presentations of infections include ventilator-associated pneumonia, bloodstream infection, wound infection and urinary tract infection. ${ }^{[1]}$ Antibiotic resistance is an important concern while treating Acinetobacter infections. Multidrug resistance (MDR) Acinetobacter spp. technically refers to non-susceptibility to $\geq 1$ agent in $\geq 3$ antimicrobial categories. In contrast, extensive drug resistance (XDR) refers to non-susceptibility to $\geq 1$ agent in all but $\leq 2$ antimicrobial categories and is more clinically relevant. Non-susceptibility to all antimicrobial agents tested defines pan-drug resistance (PDR). Resistance to carbapenem poses a major challenge as the second-line treatment options are much less defined in their efficacy and often accompany toxicity. This along with its ability to survive on dry surfaces for prolonged periods facilitates its dissemination in the healthcare environment, often leading to outbreaks. ${ }^{\text {[2] }}$

Currently, there are concerns with ampicillin/sulbactam due to increasing resistance and the dosing frequency needed to provide adequate high concentrations of sulbactam. Carbapenems used in combination have historically resulted in positive clinical outcomes, but resistance is increasing. Colistin retains susceptibility against A. baumannii, but issues of appropriate dosing, toxicities, and resistance remain. $^{[3]}$

Antimicrobials such as tigecycline, ceftolazonetazobactam and ceftazidime-avibactam have more recently come to market; but Ceftolazonetazobactam and ceftazidime-avibactam have minimal utility in the treatment of carbapenem resistant Acinetobacter baumannii (CRAB), given the usual mechanisms of resistance to carbapenems are class D or B carbapenemases. Both of these carbapenemases readily hydrolyze these cephalosporins and these enzymes are not inhibited by either tazobactam or avibactam. While tigecycline shows excellent in vitro activity against $\mathrm{CRAB}$, enthusiasm surrounding this agent has been tempered due to pharmacokinetic limitations of the drug, the development of resistance while on therapy, and its inability to demonstrate non-inferiority in the treatment of hospital-acquired and ventilator-associated pneumonia with suboptimal drug levels in the epithelial lining fluid being suggested as a possible cause for its poor performance. [4, 5] Because infections due to CRAB are most commonly seen in the lungs, blood, and urine, the role of tigecycline in the treatment of these resistant pathogens remains unclear and its efficacy uncertain. ${ }^{[5]}$

Minocycline has been proposed as a potential alternative to tigecycline given its pharmacokinetic advantage in the blood, where it achieved higher serum concentrations (2.1 to 6.6 $\mu \mathrm{g} / \mathrm{mL}$ with the $200 \mathrm{mg}$ IV dose) and its FDAapproved indication for treatment of infections due to Acinetobacter spp. as well as for urinary tract infections. Additionally, with the availability of both IV and PO minocycline formulations, and the relatively high bioavailability of the oral formulation, the ability to switch from IV to PO routes with minocycline is attractive. ${ }^{[5]}$ Minocycline being more lipophilic allows for increased tissue penetration with longer half-lives. The most common efflux pump mediating tetracycline resistance does not affect minocycline. ${ }^{[5]}$

Tigecycline was synthetically designed to overcome these efflux pumps and has activity if either TetA or TetB is present. Current literature suggests that the development of such resistance has been associated with the Tet $X$ gene and, perhaps most importantly for A. baumannii, the overexpression of various efflux pumps (AdeABC, AdeIJK, AdeFGH, AbeM, AdeDE). ${ }^{[6]}$ AdeABC, have been closely associated with the development of tigecycline resistance. Upregulation of these AdeABC pumps have been associated with higher MICs and increased resistance to tigecycline in vitro but interestingly, 
these pumps do not appear to impact minocycline susceptibility, and therefore tigecycline-resistant A. baumannii can remain susceptible to minocycline. Importantly, these RND pumps can be selected with clinically relevant concentrations of tigecycline (but not minocycline) and serve as a mechanism for the well-described development of tigecycline resistance while on therapy. ${ }^{[7]}$

A. baumannii containing oxacilinases or metalobeta-lactamase genes has been reported to retain a susceptibility profile to minocycline similar to organisms without these genes. Thus, minocycline susceptibility should be determined by testing directly and not be determined by a surrogate class approach (i.e. tigecycline and doxycycline resistance does not necessarily equal minocycline resistance). ${ }^{[7]}$

It has been hypothesized that minocycline elimination is independent of renal function and hepatic function. As minocycline does not seem to accumulate in renal failure, it is believed that minocycline can be safely administered to patients with renal insufficiency without requiring any dose adjustments. ${ }^{[4]}$

\section{Aims \& Objectives}

To determine the activity of tetracycline group of drugs against Acinetobacter species.

\section{Materials and methods}

A total of 300 non- duplicate Acinetobacter spp were included in the study. These were isolated from various samples including urine, blood, pus, endotracheal secretion, central line tips. Acinetobacter species were identified by Gram's staining, catalase test, oxidase test, motility and by using conventional sugar fermentation tests/routine biochemical tests by standard laboratory procedures. $^{[8]}$ Antimicrobial susceptibility test (amikacin, piperacillintazobactam, cefoperazone-sulbactam, polymixin B, tetracycline, doxycycline, minocycline, tigecycline) was performed by using the KirbyBauer disc diffusion techniques according to CLSI guidelines. ${ }^{[9]}$

\section{Result}

From a total of 300 Acinetobacter species isolated during the study period from January 2015 to December 2016, 61.7\% were CRAB isolates, 59.3 $\%$ were multi-drug resistant Acinetobacter spp. Acinetobacter spp simultaneously resistant to carbapenems, fluoroquinolones and beta -lactams were defined as MDR in our study. Majority of isolates were sensitive to polymixinB (95\%), minocycline $(67.3 \%)$ and tigecycline (78\%). Majority of isolates were resistant to tetracycline (77.3\%), doxycycline(70.3\%), amikacin(97\%), cefoperaone-sulbactum $(95.1 \%)$ and carbapenems (61.7\%). Majority of the isolates were from pus specimen $(26.6 \%)$ followed by urine $(22.3 \%)$, blood specimen(18.3\%), ET secretions(17\%) and central line tips $(15 \%)$. Tigecycline was the second most effective antibiotic after Colistin, followed by minocycline. Out of the 10 strains which were resistant to Colistin as well as tigecycline, the only other effective antibiotic was Minocycline (7/10). The samplewise distribution of the Acientobacter isolates has been depicted in Table 1.

Table 1: Sample wise distribution of the isolates with their antibiotic susceptibility pattern

\begin{tabular}{|c|c|c|c|c|c|}
\hline $\begin{array}{l}\text { Sample(30 } \\
\text { 0) }\end{array}$ & $\begin{array}{c}\text { Colistin } \\
(285 / 30 \\
0) \\
(95 \%)\end{array}$ & $\begin{array}{c}\text { Minocycli } \\
\text { ne } \\
(202 / 300) \\
(67.3 \%)\end{array}$ & $\begin{array}{c}\text { Tigecycli } \\
\text { ne } \\
(234 / 300) \\
(78 \%)\end{array}$ & $\begin{array}{c}\text { Doxycycli } \\
\text { ne } \\
(89 / 300) \\
(29.7 \%)\end{array}$ & $\begin{array}{c}\text { Tetracycli } \\
\text { ne } \\
(68 / 300) \\
(22.7 \%)\end{array}$ \\
\hline Urine (67) & $\begin{array}{c}66 \\
(98.5 \%)\end{array}$ & $46(68.6 \%)$ & $53(79 \%)$ & $15(22.5 \%)$ & $10(14.9 \%)$ \\
\hline Blood (55) & $\begin{array}{c}52 \\
(94.5 \%)\end{array}$ & $36(65.4 \%)$ & $\begin{array}{c}41 \\
(74.5 \%)\end{array}$ & $16(29 \%)$ & $11(20 \%)$ \\
\hline Pus (80) & $\begin{array}{c}75 \\
(93.5 \%)\end{array}$ & $61(76 \%)$ & $\begin{array}{c}67 \\
(83.7 \%) \\
\end{array}$ & $\begin{array}{c}25 \\
(31.3 \%)\end{array}$ & $20(31.3 \%)$ \\
\hline $\begin{array}{l}\text { ET } \\
\text { secretions } \\
(53)\end{array}$ & $\begin{array}{c}50 \\
(94.3 \%)\end{array}$ & $34(64.1 \%)$ & $\begin{array}{c}37 \\
(69.8 \%)\end{array}$ & $\begin{array}{c}24 \\
(45.3 \%)\end{array}$ & $21(45.3 \%)$ \\
\hline \begin{tabular}{l}
\multicolumn{2}{l}{ Central } \\
line tips \\
$(45)$
\end{tabular} & $\begin{array}{c}42 \\
(93.3 \%)\end{array}$ & $25(55.5 \%)$ & $36(80 \%)$ & $9(20 \%)$ & $6(20.1 \%)$ \\
\hline
\end{tabular}

\section{Discussion}

This study highlights that minocycline was highly effective in CRAB isolates which is similar to the study by Castanheira et al. who demonstrated that the only two antimicrobials with greater than $50 \%$ susceptibility rates were minocycline and colistin. ${ }^{[10]}$ Reports of minocycline retaining its activity in patient isolates that have developed colistin resistance during colistin therapy also 
exist. Importantly, minocycline susceptibility remains high and is not impacted by carbapenem resistance. Additionally, it is important to note that minocycline has also displayed high levels of synergistic activity with polymyxins in minocycline-resistant isolates, where $0.5 \mathrm{mg} / \mathrm{L}$ of Colistin restored in vitro susceptibility to minocycline in isolates, which were otherwise resistant to minocycline monotherapy. This is an important and encouraging finding given the propensity to use combination regimens in the treatment of CRAB. ${ }^{[4]}$

In our study tigecycline was the second most active agent, following Colistin but most of the isolates were from blood cultures, central line tips from suspected cases of catheter related blood stream infections and ET secretions. Many studies have pointed out the doubtful effectiveness of tigecycline in blood and pulmonary sites, making minocycline as probably the most effective antibiotic after Colistin. Castanheira et al. in his study also found minocycline was the second most active (79.1\% susceptible) agent, only exceeded by colistin ( $98.8 \%$ susceptibility using current breakpoints of $\leq 2 \mu \mathrm{g} / \mathrm{mL}){ }^{[8]}$

Although minocycline was found to be resistant to a large number of cases other studies have proposed that empirical therapy including recommended combination therapy is IV minocycline plus IV colistin for the treatment of A. baumannii infections ${ }^{[3]}$, which also helps in circumventing the side effects of colistin.

The study highlights that the $\beta$ lactam + sulbactam combinations have practically no activity against Acinetobacter species. Even though Colistin was the most effective antibiotic, minocycline was the only effective antibiotic against the Colistin resistant strains

Currently, there are concerns with ampicillin/ sulbactam due to increasing resistance and the dosing frequency needed to provide adequate high concentrations of sulbactam. Carbapenems used in combination have historically resulted in positive clinical outcomes, but resistance is increasing showing a susceptibility of $38.3 \%$ in our study.
Colistin retains susceptibility against $A$. baumannii, but issues of appropriate dosing, toxicities, and resistance remain. Minocycline represents an attractive option in the treatment of MDR-AB infections as susceptibility to isolates form blood cultures, respiratory sites where the activity of tigecycline is doubtful, remains high.

The clinical studies evaluating the use of minocycline for the treatment of ventilatorassociated pneumonia caused by MDR A. baumannii, cures were achieved for $80.6 \%-86.0 \%$ of the patients receiving minocycline-based treatments. ${ }^{[10]}$ These independent investigations showed that minocycline might be valuable choices for the treatment of this high-mortality infection when other agents are not active or are inappropriate. In the study by Debra et al, no patient had any documented adverse events deemed to be attributable to minocycline. ${ }^{[3]}$

The study shows that despite the organism showing resistance to other tetracyclines the in vitro efficacy of minocycline was not affected, which is important from the microbiologists point of view to look for minocycline sensitivity despite the organism being resistant to even tigecyclines.

\section{References}

1. Peleg AY, Seifert H, Paterson DL. Acinetobacter baumannii: emergence of a successful pathogen. Clin Microbiol Rev. Jul; 2008 21(3):538-582.

2. Viehman JA, Nguyen MH, Doi Y. Treatment Options for CarbapenemResistant and Extensively Drug-Resistant Acinetobacter baumannii Infections. Drugs. 2014; 74(12): 1315-1333

3. Debra A. Goff, Karri A. Bauer, Julie E. Mangino. Bad Bugs Need Old Drugs: A Stewardship Program's Evaluation of Minocycline for Multidrug-Resistant Acinetobacter baumannii Infections. Clin Infect Dis. 2014;59(6):S381-S387

4. Lashinsky JN, Henig O, Pogue JM, Kaye KS. Minocycline for the Treatment of Multidrug and Extensively Drug-Resistant 
A. baumannii: A Review. Infect Dis Ther. 2017;6(2):199-211.

5. Jason M. Pogue, Anupama Neelakanta, Ryan P. Mynatt, Sarit Sharma, Paul Lephart, Keith S. Kaye; CarbapenemResistance in Gram-Negative Bacilli and Intravenous Minocycline: An Antimicrobial Stewardship Approach at the Detroit Medical Center, Clin Infect Dis 2014;59(6):S388-S393.

6. Pournaras S, Koumaki V, Gennimata V, Kouskouni E, Tsakris A. In vitro activity of tigecycline against Acinetobacter baumannii: global epidemiology and resistance mechanisms. Adv Exp Med Biol.2016;897:1-14.

7. Rizek C, Ferraz JR, van der Heijden IM, Giudice M, Mostachio AK, Paez J, et al. In vitro activity of potential old and new drugs against multidrug-resistant gramnegatives. J Infect Chemother. 2015;21(2):114-7.

8. Collee JG, Fraser AG, Marmion BP, Simmons A, editors. Mackie \& McCartney Practical Medical Microbiology. India: Elsevier; 2012.

9. Clinical and Laboratory Standards Institute. 2016. Performance standards for antimicrobial susceptibility testing, 26th ed. CLSI supplement M100S. Clinical and Laboratory Standards Institute, Wayne, PA.

10. Castanheira M, Rodrigo E. Mendes, Ronald N. Jones; Update on Acinetobacter Species: Mechanisms of Antimicrobial Resistance and Contemporary In Vitro Activity of Minocycline and Other Treatment Options, Clin Infect Dis.2014;59(6):S367-S373. 